increases care needs. We aimed to evaluate the impact of a hypothetical cognitive rehabilitation intervention on PSCI outcomes using the StrokeCog epidemiological model.

Methods We developed a probabilistic Markov model to project and track incidence and prevalence of PSCI in the Irish population aged 40-89 years to 2035. Data sources included official population and hospital episode statistics, and longitudinal cohort studies. Drawing on available systematic review evidence, we hypothesized that cognitive rehabilitation would reduce the risk of cognitive impairment no dementia (CIND) at 1 year post-stroke by $18 \%$ (scenario $1, \mathrm{~S} 1$, small effect) or by $54 \%$ (scenario $2, \mathrm{~S} 2$, medium effect) relative to usual care.

Results In usual care, the projected prevalence of post-stroke CIND in Ireland in 2035 was 6.7 per 1000 general population $(95 \%$ CI $5.6-7.8)$, or $35 \%$ of stroke survivors $(95 \% \mathrm{CI}$ $30.5-38.8) \quad(n=21026$ prevalent cases). In S1 (small effect) the projected prevalence was reduced to $32.0 \%$ (95\% CI 28.6$36.4)$ of stroke survivors $(n=19652)$, and in S2 (medium effect) to $29.1 \%(95 \%$ CI $25.2-33.2)$ of stroke survivors $(n=17672)$. The number of years of life lived free of cognitive impairment were increased by $6.3 \%$ in S1 (small effect) and $15.1 \%$ in S2 (medium effect).

Conclusion The StrokeCog model provides a tool for policymakers and researchers to evaluate the potential impact of cognitive rehabilitation at different levels of intervention effectiveness. The model was based on conservative assumptions, and a less conservative approach could lead to a greater projected reduction in burden. Our next steps include analysis of quality of life outcomes and costs.

\section{OP76 CHRONIC KIDNEY DISEASE IN CHILE: FINDINGS FROM THE CHILEAN NATIONAL HEALTH SURVEYS 2009-10 AND 2016-17}

${ }^{1} \mathrm{M}$ Walbaum, ${ }^{1} \mathrm{~S}$ Scholes, ${ }^{2} \mathrm{E}$ Pizzo, ${ }^{3} \mathrm{M}$ Paccot, ${ }^{1} \mathrm{JS}$ Mindell ${ }^{*} .{ }^{1}$ Health and Social Surveys Research Group, Research Department of Epidemiology and Public Health, University College London, London, UK; ${ }^{2}$ Department of Applied Health Research, University College London, London, UK; ${ }^{3}$ Department of Non-communicable diseases, Ministry of Health of Chile, Santiago, Chile

\subsection{6/jech-2020-SSMabstracts.75}

Background Chronic kidney disease (CKD) is a leading global public health problem, with a substantial burden on healthcare systems; decreased quality of life, and poor prognosis for patients. In Chile, there is limited data on CKD prevalence and its distribution across population subgroups that impedes effective decision-making in the healthcare sector. The objectives were to estimate the prevalence of CKD among Chilean adults and examine its associations with sociodemographic characteristics, health behaviours, and comorbidities.

Methods Analysis of cross-sectional data from the two most recent large, nationally-representative Chilean Health Surveys (ENS) 2009-10 and 2016-17. The participants were individuals aged $15+$ years with serum creatine data (ENS 2009-10: $n=4777$; ENS 2016-17: $n=5279$ ). The primary outcome was reduced kidney function (CKD Stages 3a-5) based on estimated glomerular filtration rate (eGFR $<60$ $\left.\mathrm{mL} / \mathrm{min} / 1.73 \mathrm{~m}^{2}\right)$. Increased albuminuria $(\geq 30 \mathrm{mg} / \mathrm{g})$, the secondary outcome measure, was determined using the urine albumin-to-creatinine ratio (ACR) ascertained among adults aged $40+$ years with diabetes and/or hypertension. Both outcomes were analysed using logistic regression and the combined two-survey dataset, with results summarised using odds ratios (OR). CKD prevalence (Stages 1-5) among adults aged $40+$ years was estimated using an expanded definition including participants with a reduced eGFR or an eGFR of at least $60 \mathrm{~mL} / \mathrm{min} / 1.73 \mathrm{~m}^{2}$ but increased albuminuria (Stages 1-2). Analyses were adjusted for non-response and complex survey design.

Results Overall, 3.0\% (95\% CI: 2.4-3.8\%) of adults in ENS 2016-17 had reduced kidney function. After full adjustment, participants with hypertension (OR 2.12; 95\% CI 1.08-4.16) and those with diabetes (OR 1.66; 1.04-2.65) had significantly higher odds of reduced kidney function. 15.5\% (13.5$17.8 \%$ ) of adults aged $40+$ years with diabetes and/or hypertension had increased albuminuria in 2016-17. Being obese versus normal weight (OR 1.66; 1.08-2.54) and having both diabetes and hypertension versus diabetes alone (OR 2.30; 1.34-3.95) were significantly associated with higher odds of increased albuminuria in fully-adjusted analyses. At least $15.4 \%$ of all adults aged $40+$ in ENS 2016-17 had CKD (Stages 1-5) according to the expanded definition, including 9.6\% of adults with CKD Stages 1-2.

Conclusion There is a high prevalence of Chilean adults at CKD Stages 1-2 that should be considered in the prevention strategies and Chilean healthcare guidelines.

\section{OP77 THE PREVALENCE AND CHARACTERISTICS OF ADVERSE DRUG REACTION-RELATED HOSPITAL ADMISSIONS IN OLDER PATIENTS}

${ }^{1} \mathrm{CC}$ Cahir*, ${ }^{2} \mathrm{CC}$ Curran, ${ }^{1} \mathrm{CW}$ Walsh, ${ }^{2} \mathrm{DW}$ Williams, ${ }^{1} \mathrm{~KB}$ Bennett. ${ }^{1}$ Division of Population Health Sciences, Royal College of Surgeons in Ireland, Dublin, Ireland; ${ }^{2}$ Department of Geriatric and Stroke Medicine, Royal College of Surgeons in Ireland and Beaumont Hospital, Dublin, Ireland

\subsection{6/jech-2020-SSMabstracts.76}

Background Older people experience greater morbidity with a corresponding increase in medication use resulting in a potentially higher risk of adverse drug reactions (ADRs).The aim of this study was to determine the prevalence and characteristics of ADR-related hospital admissions among older patients $(\geq$ 65 years).

Methods A cross-sectional study of ADR prevalence in patients aged $\geq 65$ years admitted acutely to a large tertiary referral hospital in Ireland over a 7 month period (November 2016June 2017). A multifaceted review of each hospital admission was undertaken to assess the likelihood of an ADR being a reason for admission (cause of admission or contributing to admission) in the context of the patient's medication, clinical condition, medical history, comorbidities and investigations. A number of decision aids were also applied by two independent reviewers to assess ADR causality (Naranjo criteria, WHO criteria, Liverpool Algorithm). The avoidability (Hallas criteria) and severity (Hartwig severity assessment scale) of the ADR were also assessed. Differences in causality, preventability and severity were reviewed by a third reviewer.

Results In total, 3760 hospital admission episodes (in 3091 patients) were screened and 377 admissions were ADR-related (10.02\%, 95\% CI 9.06\%, 10.98\%); 43 admissions were due to $\geq 2$ ADRs ( $\mathrm{N}=424$ ADRs). 360 (11.64\% 95\% CI 10.51\%, $12.77 \%)$ patients had at least one ADR with $50(16.18 \%)$ patients experiencing $\geq 1$ ADR-related admission. In summary, 
219 (58.09\%) admissions were caused by an ADR, while ADRs contributed to 158 (41.91\%) admissions. For the majority of admissions $(\mathrm{N}=216,57.60 \%)$ there was no other known acute medical issue that may have acted in synergy with the medication resulting in the ADR. Of the 377 ADRrelated admissions, 43 (11.41\%) were deemed definitely avoidable and 225 (59.68\%) possibly avoidable and 350 (92.84\%) were classified as moderate severity.

Conclusion One in ten hospital admissions were ADR-related, with approximately $70 \%$ potentially avoidable. Interventions are needed to reduce ADRs and improve medication management in older populations.

\section{OP78 PROJECTION OF THE ECONOMIC AND HEALTH IMPACTS OF CHRONIC KIDNEY DISEASE IN THE CHILEAN POPULATION}

${ }^{1} \mathrm{M}$ Walbaum*, ${ }^{1} \mathrm{~S}$ Scholes, ${ }^{2} \mathrm{E}$ Pizzo, ${ }^{3} \mathrm{R}$ Rojas, ${ }^{1} \mathrm{JS}$ Mindell. ${ }^{1}$ Health and Social Surveys Research Group, Research Department of Epidemiology and Public Health, University College London, London, UK; ${ }^{2}$ Department of Applied Health Research, University College London, London, UK; ${ }^{3}$ School of Health and Related Research, University of Sheffield, Sheffield, UK

\subsection{6/jech-2020-SSMabstracts.77}

Background Chronic Kidney Disease (CKD) is a leading public health problem, with substantial burden on and economic implications for healthcare systems, mainly from renal replacement treatment (RRT) for End-Stage Kidney Disease (ESKD). In Chile, the prevalence of CKD Stages 1 to 5 in people aged 40 years or older is at least $15.4 \%$, with an increasing rate of adults receiving RRT. The aim of this study is to develop a model to estimate the future burden of the disease in Chile, given the high and rising prevalence of comorbidities for CKD.

Methods A dynamic deterministic Markov model was developed to simulate CKD in the Chilean population aged $40+$ from the perspective of the Chilean public healthcare system, up to the year 2070. Key parameters: prevalence of CKD by stages, prevalence of hypertension and diabetes, mortality rate and direct costs of treatment, were extracted from nationallyrepresentative Chilean data. Incidence of the disease and progression rates were simulated based on the most relevant international evidence. The model included seven states replicating the natural progression of the disease: from CKD Stage 2 through to Stage 5, ESKD with need of RRT, and death. The progression of CKD was assumed in 1-year cycles and was categorised as slow and fast progression (decrease in the estimated glomerular filtration rate of $3 \mathrm{ml} / \mathrm{min} /$ year or $8 \mathrm{ml} /$ $\mathrm{min} /$ year, respectively). At the end of each cycle, a proportion of individuals remained in the same state, progressed to the next CKD stage, began RRT or died. We calibrated the model based on international evidence and conducted one-way sensitivity analyses by varying key model parameters to create different scenarios. We used Stata V15.1 (StataCorp, College Station, Texas, USA) to estimate the model parameters and Microsoft Excel Office 365 V2001 was used to construct the Markov model.

Results By the year 2070, there is an expected increase in the number of adults with ESKD, ceteris paribus, from 22,300 to 58,000 people, with an expected increase in direct costs of CKD stages $2-5$ from $£ 213$ million to $£ 506$ million. A reduction in the percentage of fast progressors could reduce the total cases of ESKD to 42,933 and the expected direct costs by around $£ 110$ million.

Conclusion The estimates in this study show an important increase in the cases and costs of CKD. This model can be a useful tool for healthcare planning, with development of preventive or treatment plans to reduce and delay the progression of the disease.

\section{Friday 11 September}

\section{Interventions}

\section{OP79 OPTIMISING PATIENT AND PUBLIC ENGAGEMENT IN TRIALS OF COMPLEX INTERVENTIONS}

N Kime*, A Heaven, C Quinn, S Brown. Academic Unit of Ageing and Stroke Research, Bradford Institute for Health Research, Bradford, UK

\subsection{6/jech-2020-SSMabstracts.78}

Background Public involvement is mandated by the National Institute for Health Research (NIHR) and its 'UK Standards for Public Involvement in Research' provide a benchmark for effectiveness. Whilst guidance exists about how, when and why to enact public involvement, there is still debate around how to evaluate it and its impact. Public involvement is an integral component of the personalised care planning for older people with frailty programme (PROSPER). This work aimed to evaluate public involvement structures in the feasibility stage of the PROSPER programme and provide recommendations for its optimisation.

Methods Two lay representatives with requisite training and skills conducted qualitative face-to-face interviews with lay members and stakeholders from all levels of the PROSPER programme, from strategic to operational. The interviews explored influence and impact. In addition, documentary analysis of minutes from meetings involving lay members was performed to identify to what extent lay member involvement was embedded in the Programme along with barriers and facilitators to engagement. Two researchers and the lay representatives conducted thematic analysis of both sets of data using a framework based on the NIHR standards.

Results Twelve qualitative interviews and twenty three documentary analyses of meeting minutes were conducted. Identified themes were centred on: 1) individual members, notably training and skills utilisation and development and learning around the research process, and 2) the demonstrable impact of public involvement on individuals, the Programme, NHS research and the wider community. Improvements to current arrangements, including diversity of representation in the public involvement groups and the timely communication of information to its members, were proposed as recommendations for the development of public involvement in the definitive trial.

Conclusion This evaluation has shown that the current public involvement structures in PROSPER largely meet the NIHR standards. Furthermore, it has highlighted tangible impacts of public involvement for individuals, the Programme and the wider community. However, several areas of improvement were recognised which will be addressed in the definitive trial. Strengths and limitations around lay representatives conducting interviews and members' impact on the research were identified. 